\title{
Construction of a novel immune- related IncRNA signature and its potential to predict the immune status of patients with hepatocellular carcinoma
}

\author{
Min Deng ${ }^{1,2,3 \dagger}$, Jia-Bao Lin ${ }^{4 \dagger}$, Rong-Ce Zhao ${ }^{1,2,3 \dagger}$, Shao-Hua Li ${ }^{1,2,3}$, Wen-Ping Lin ${ }^{1,2,3}$, Jing-Wen Zou ${ }^{1,2,3}$,
} Wei Wei ${ }^{1,2,3}$ and Rong-Ping Guo ${ }^{1,2,3^{*}}$ (1)

\begin{abstract}
Background: The accuracy of existing biomarkers for predicting the prognosis of hepatocellular carcinoma (HCC) is not satisfactory. It is necessary to explore biomarkers that can accurately predict the prognosis of HCC.

Methods: In this study, original transcriptome data were downloaded from The Cancer Genome Atlas (TCGA) database. Immune-related long noncoding ribonucleic acids (irlncRNAs) were identified by coexpression analysis, and differentially expressed irlncRNA (DEirlncRNA) pairs were distinguished by univariate analysis. In addition, the least absolute shrinkage and selection operator (LASSO) penalized regression was modified. Next, the cutoff point was determined based on the area under the curve (AUC) and Akaike information criterion (AIC) values of the 5-year receiver operating characteristic $(\mathrm{ROC})$ curve to establish an optimal model for identifying high-risk and low-risk groups of HCC patients. The model was then reassessed in terms of clinicopathological features, survival rate, tumorinfiltrating immune cells, immunosuppressive markers, and chemotherapy efficacy.
\end{abstract}

Results: A total of 1009 pairs of DEirlncRNAs were recognized in this study, 30 of these pairs were included in the Cox regression model for subsequent analysis. After regrouping according to the cutoff point, we could more effectively identify factors such as aggressive clinicopathological features, poor survival outcomes, specific immune cell infiltration status of tumors, high expression level of immunosuppressive biomarkers, and low sensitivity to chemotherapy drugs in HCC patients.

Conclusions: The nonspecific expression level signature involved with irlncRNAs shows promising clinical value in predicting the prognosis of HCC patients.

Keywords: Hepatocellular carcinoma, Immune-related IncRNA, Prognosis, Bioinformatics

*Correspondence: guorp@sysucc.org.cn

${ }^{\dagger}$ Min Deng, Jia-Bao Lin and Rong-Ce Zhao contributed equally to this work.

${ }^{3}$ Collaborative Innovation Center for Cancer Medicine, 651 Dongfeng East Road, Guangzhou, China

Full list of author information is available at the end of the article

\section{Background}

Hepatocellular carcinoma (HCC) is the fourth most common malignant tumor globally, and its incidence is increasing annually with poor survival $[1,2]$. The primary relevant risk factors associated with the development of HCC include viral hepatitis, alcoholic liver disease, nonalcoholic fatty liver disease, aflatoxin exposure [3]. In recent years, chemotherapeutics have achieved 
encouraging results in treating HCC, especially immune checkpoint inhibitors (ICIs) [4]. With the recent success of clinical trials of immunotherapy, such as Checkmate 040, Keynote-224, and IMbrave150, ICIs such as nivolumab, pembrolizumab, and atezolizumab plus bevacizumab have been approved for the treatment of $\mathrm{HCC}$ [5-7].

Long noncoding RNAs (lncRNAs) are nonproteincoding RNAs with a transcription length of more than 200 nucleotides [8]. Because lncRNAs are abundant, they often participate in human physiological processes and are closely related to the development of diseases [9-11]. In addition, lncRNAs have the ability to interact with molecules such as DNA, RNA, or protein to play enhancing or inhibitory roles [12]. Studies have reported that lncRNAs may participate in tumorigenesis through various molecular mechanisms [13, 14]. Recent work has demonstrated that lncRNAs can promote the malignant phenotypes of cancer by changing the genome or transcriptome level and varying the immune microenvironment [15]. LncRNAs can activate immune cells by expressing related genes, which leads to immune cells infiltrating tumors [16].

Immune cell infiltration markers in tumors show prospective predictive and prognostic value for tumor diagnosis, treatment, and survival evaluation [17-19]. Moreover, because lncRNAs have a close relationship with tumor immunity, the study of IncRNAs in combination with tumor immunity will help to establish these markers. The researches of Hong [20], Wei [21], and $\mathrm{Qu}[20]$ constructed models to predict the prognosis of HCC, pancreatic cancer, and clear cell renal cell carcinoma based on the immune-related lncRNAs (irlncRNAs) and risk scores, which have certain accuracy in predicting the prognosis of tumor patients. Our study built a novel signature constructed by irlncRNAs to predict HCC patients' prognosis. IrlncRNAs, such as LINC01138, THUMPD3-AS1, AL365203, TBX2-AS1, have been confirmed to be related to the prognosis of HCC patients [21-24]. LINC01138 can promote cell proliferation, tumor invasion, metastasis and enhance protein stability by interacting with arginine methyltransferase 5 (PRMT5) [21]. TBX2 hypermethylation was associated with increased HCC risk [24]. While AC092535, FAM99A, AL355802, etc., have not been reported in HCC.

Generally, the accuracy of a tumor prediction model based on the combination of two biomarkers is better than that composed of a single gene [25]. To date, few models have been used to study the predictive role of lncRNAs and tumor immune-related cells in HCC [26, 27]. This study used a novel modeling algorithm, which does not require specific expression-level data, through pairing and iteration to establish an irlncRNA signature. Subsequently, we evaluated the diagnostic effect, predictive value, immune cell infiltration into tumors, and chemotherapy efficacy of this signature in HCC patients.

\section{Methods \\ Data collection and expression analysis}

The transcriptome data (RNA-seq) used for analysis in this study, corresponding to fragments per kilobase million (FPKM), was downloaded from the TCGA database (https://tcga-data.nci.nih.gov/tcga/). The GTF files used for subsequent analysis, distinguishing mRNA and lncRNA, were extracted from the genome database Ensembl (http://asia.ensembl.org). The ImmPort database (http://www.immport.org) was accessed to download a list of identified immune-related genes (ir-genes), serving a screening role to filter irlncRNAs through coexpression methods [28, 29]. The correlation between lncRNAs and ir-genes was analyzed. The inclusion criteria of the irlncRNAs were an immune gene correlation coefficient $>0.4$ and a $p$-value $<0.001$. The limma package of $\mathrm{R}$ was used to analyze the differential expression among irlncRNAs, and a log fold change $(\mathrm{FC})>1$ and false discovery rate $(\mathrm{FDR})<0.05$ were regarded as thresholds to distinguish different irlncRNAs (DEirlncRNAs).

\section{DEirlncRNA pairing}

DEirlncRNAs were periodically single paired. When $C$ was assumed to be equal to lncRNA A plus lncRNA B, a 0 or 1 matrix was constructed. When the lncRNA A expression level was higher than that of IncRNA B, C was defined as 1 ; otherwise, $C$ was defined as 0 . Then, the constructed matrix was further screened. When the expression level of lncRNA pairs was assumed to be 0 or 1 , no relationship between pairing and prognosis was presumed because there was no specific level of pairing to correctly predict the survival outcome of patients [28, 29]. When the number of lncRNA pairs with an expression of 0 or 1 accounted for more than $30 \%$ of the total pairs, it was regarded as an effective match.

\section{Clinicopathological data acquisition}

The clinicopathological information of HCC patients was collected from the TCGA database. After excluding cases with follow-up times less than 30 days and duplicate data, adequate data were extracted. Patients with tumor stage I (164 cases), stage II (77 cases), stage III (81 cases), and stage IV ( 3 cases) were recruited in this study.

\section{Establishing a risk model for evaluating the riskScore}

The single-factor analysis was the first step in model generation. Then, LASSO regression with 10 -fold cross-validation was carried out, and the $p$-value was 0.05 . LASSO 
regression was performed for 1000 cycles, and 1000 random stimulations were set in each process. The next step was to record the frequency of each pair in the LASSO regression model, which was repeated 1000 times, and the pairs with a frequency greater than 100 times were selected for Cox proportional hazard regression analysis and model generation. The area under the curve (AUC) value was calculated, and the curve was plotted. When the maximum AUC value is that of the highest point of the curve, the calculation process is terminated, and the model is regarded as the best candidate [28, 29]. In this study, 1-year, 3-year, and 5-year receiver operating characteristic (ROC) curves of the risk model were drawn. The formula of the risk score for all clinical cases is as follows.

\section{RiskScore $=\Sigma_{i=1}^{k} \beta i S i$}

The Akaike information criterion (AIC) value of each point of the 5-year ROC curve was used to determine the cutoff point to distinguish the high or low risk of risk scores.

\section{Risk model validation}

Kaplan-Meier analysis was used to show the difference in survival rate between the high-risk group and the lowrisk group. Then, the survival curve was plotted, and $R$ was used to visualize the risk score of each case in the model. The $\mathrm{R}$ packages glmnet, survival, survivalROC, pbapply, surfminer, and pheatmap were utilized in these analyses. A chi-square test was conducted to investigate the relationship between the clinicopathological features and the model to validate the clinical application usefulness of the generated model $[28,29]$. Then, the band chart was visualized and marked as follows: $<0.05$ was marked *, $<0.01$ was marked $* *$, and $<0.001$ was marked ***. Wilcoxon signed-rank test was performed to analyze the differences in riskScores between various clinicopathological feature groups. The results were demonstrated with box plots. Univariate and multivariate Cox regression analyses were of the risk score and clinicopathological features were performed to verify the possibility of an independent predictor of clinical prognosis for this model. The results are displayed in a forest plot. $\mathrm{R}$ packages, including ggupbr, survival, and pHeatmap, were used in these procedures.

\section{Study on tumor-infiltrating immune cells}

The currently accepted methods for examining the immune cell infiltration status between samples from the LIHC of TCGA dataset were used to analyze the relationship between risk and immune cell features. These methods included XCELL, TIMER, QUANTISEQ, MCPCOUNTER, EPIC, CIBERSORT-ABS, and CIBERSORT.
The infiltrating immune cell content analysis was followed by a comparison between the high-risk and lowrisk groups was based on Wilcoxon signed-rank test. The results are shown in a box diagram. The relationship between infiltrating immune cells and riskScores was determined by Spearman correlation analysis. A lollipop chart was drawn to show the correlation coefficient of the results $[28,29] . P<0.05$ was considered to be a significant threshold. The Ggplot2 package in R was used to perform the procedure.

\section{The noteworthy relationship between the model and the clinical therapeutics}

The IC50 of commonly used chemotherapy drugs in the LIHC dataset of the TCGA was calculated to assess the clinical application value of the model in the treatment of HCC. Chemotherapeutic medications such as bleomycin, doxorubicin, erlotinib, gemcitabine, methotrexate, mitomycin, paclitaxel, rapamycin, cisplatin, and sorafenib are commonly used in the treatment of various types of malignant tumors, as recommended by the AJCC recommendations [28, 29]. Wilcoxon signed-rank test was performed to assess IC50 between the two groups. The ggplot 2 and $\mathrm{pRRophetic} \mathrm{packages} \mathrm{in} \mathrm{R}$ were used to show the results.

\section{Expression analysis of immunosuppressive molecules related to ICls}

The relationship between the model and the gene expression level associated with ICIs was examined and visualized with a ggstatsplot and violin plot.

\section{Results}

\section{Differential expression analysis of irlncRNAs}

We downloaded transcriptome data of HCC from the TCGA database, including 50 nontumor tissues and 374 tumor tissues. The gene transfer format (GTF) files from Ensemble were used to annotate the accessed data. In total, 740 irlncRNAs were identified, of which 490 were classified as DEirlncRNAs. Among the DEirlncRNAs, 16 were downregulated, and 474 were upregulated (Fig. 1A, B, Tables S1, S2).

\section{DEirlncRNA pair screening and risk model construction}

A total of 10,344 valid DEirlncRNA pairs from the 490 DEirlncRNAs were identified by iteration loop and 0 or 1 matrix. A total of 1009 DEirlncRNA pairs were screened by a single factor test and modified LASSO analysis, of which 30 pairs were involved in the Cox model, as determined by the stepwise method. The results are shown in Fig. 1C, D, and E. Then, the AUC for each of the 1009 receiver operating characteristic (ROC) curves was calculated, and the curve was 

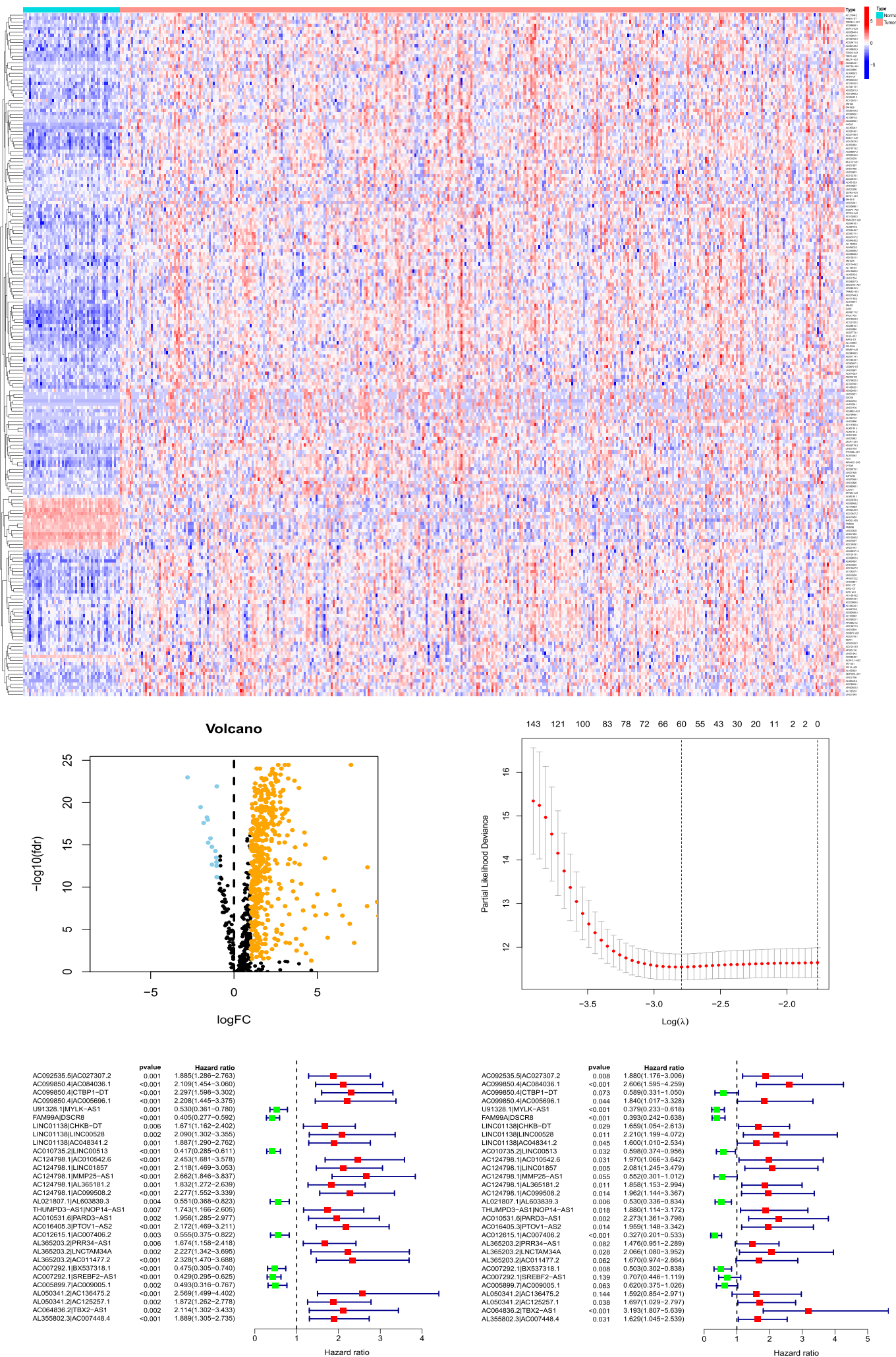

Fig. 1 Establishment of a risk assessment model based on DEirlncRNA pairs. Differentially expressed immune-related IncRNAs (DEirlncRNAs). A heat map $(\mathbf{A})$ and volcano plot $(\mathbf{B})$ are displayed. Establishment of the LASSO regression $(\mathbf{C})$. Thirty DEirlncRNA pairs are shown in a forest plot (D and $\mathbf{E})$ 


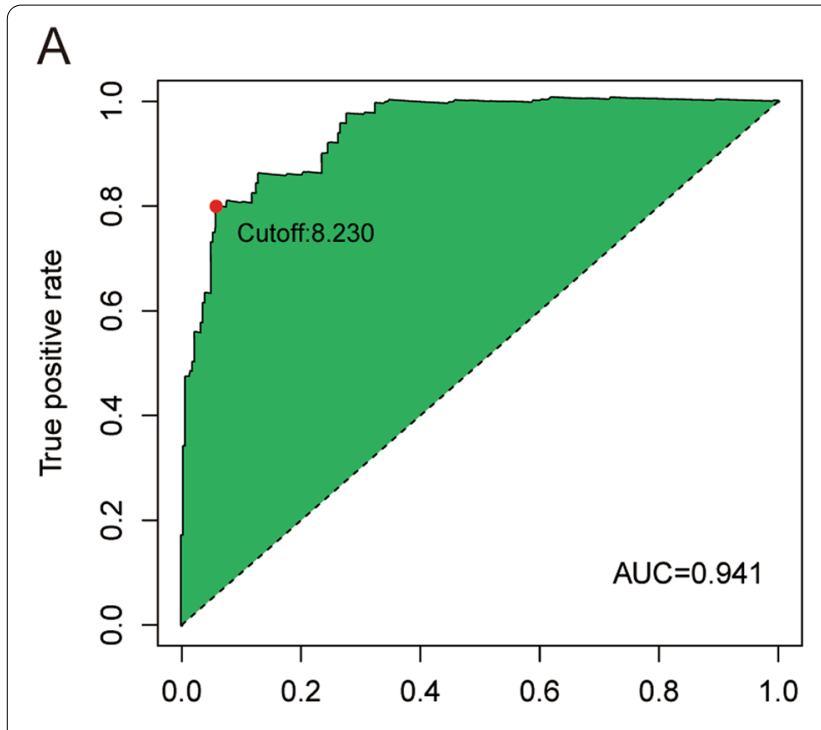

False positive rate

C

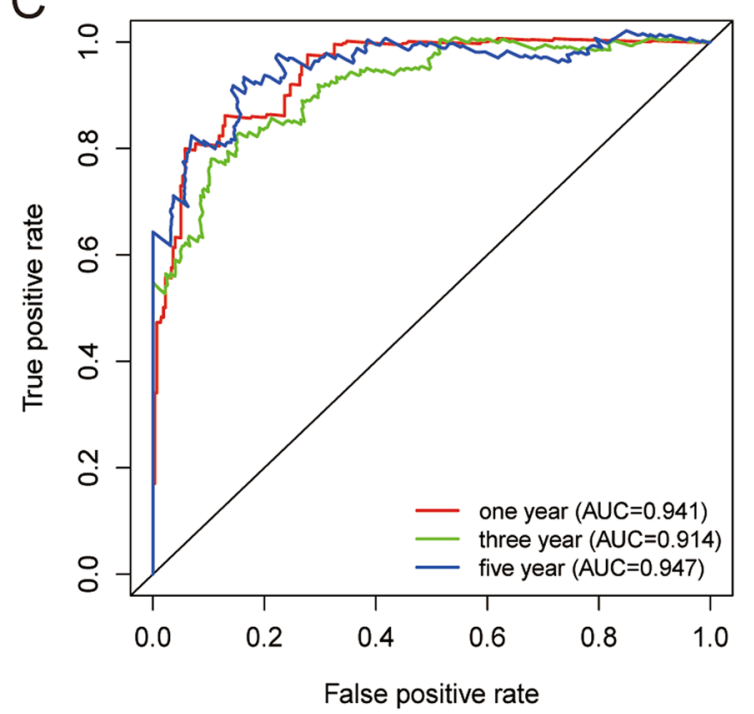

B

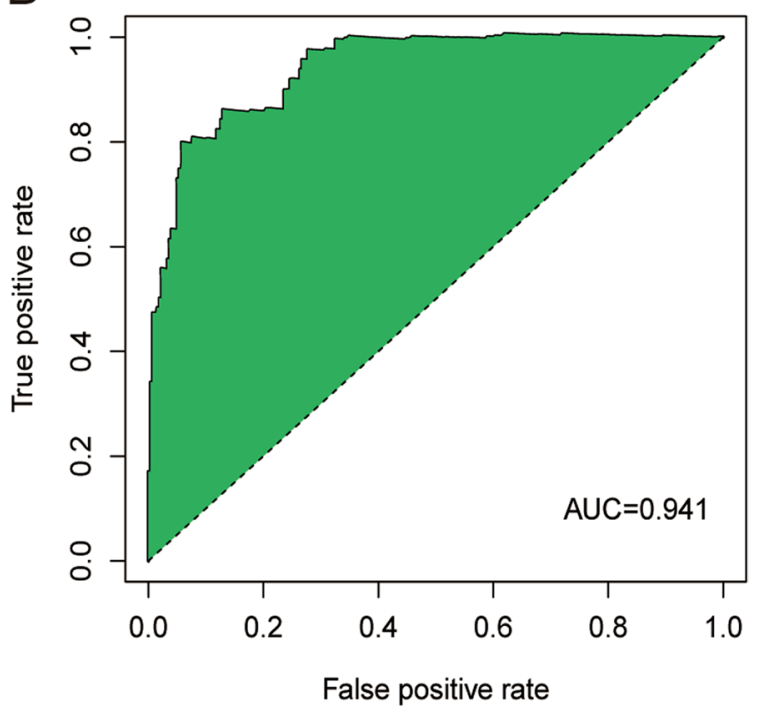

D

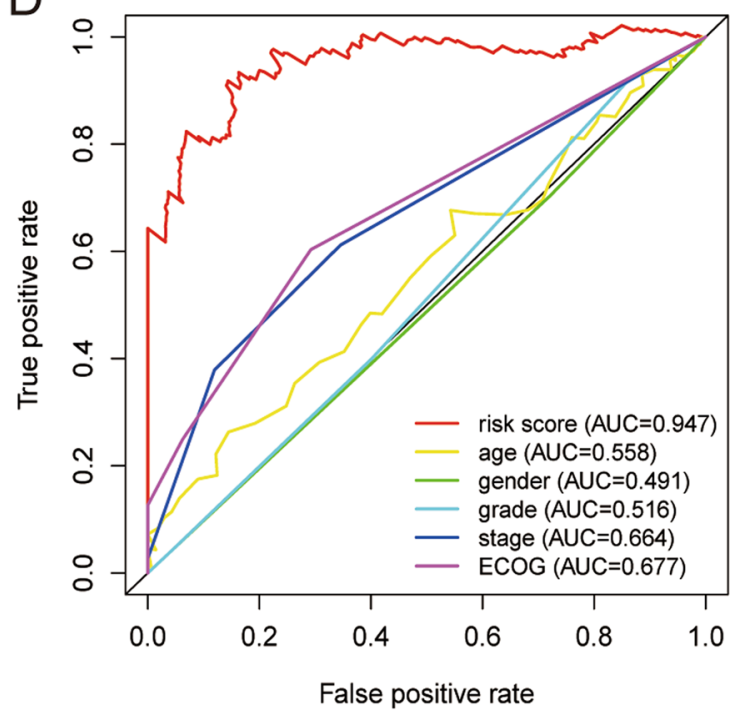

Fig. 2 Establishment of a risk assessment model on the basis of DEirlncRNA pairs. The curve of each AUC value generated by the ROCs of 1009 DEirlncRNA pair models was drawn, and the highest point of AUC was determined. The maximum inflection point was the cutoff point acquired from the AIC. A and B The 1-year, 3-year, and 5-year ROC curves of the optimal model showed that all AUC values exceeded 0.91 (C). Compared with other common clinical features, the 5-year ROC curves showed a superior risk score (D)

plotted. In addition, we found that the maximum AUC value was obtained when the highest point was equal to 0.941 , and then the optimal DEirlncRNA pair was determined (Fig. 2A). Our study also used Akaike information criterion (AIC) values to determine the maximum inflection point as the cutoff point of the 5-year ROC curve (Fig. 2B). ROC curves at 1,3 , and 5 years were drawn to verify the optimality, which indicated that all AUC values exceeded 0.91 (Fig. 2C). Moreover, AUC values between the 5-year ROC curve and some common clinical parameters were also compared. (Fig. 2D). Furthermore, data of 343 patients with HCC were collected from the TCGA database, and the risk scores of all these patients were calculated. Then, the cutoff point was utilized to redifferentiate the high-risk group and low-risk group for verification. 


\section{Application of the risk assessment model to a clinical evaluation}

Fifty-nine cases and 284 cases were divided into the highrisk and low-risk groups according to the cutoff point. Figure 3A and B display the risk scores and survival rate of each case. These data indicate that patients' clinical outcomes in the high-risk group were inferior to those in the low-risk group. The survival of the high-risk group was poorer than that of the low-risk group, as determined by Kaplan-Meier analysis $(p<0.001)$ (Fig. 3C). Next, a chi-square test was performed to explore the relationship between the risk of HCC and clinicopathological features. The stripping diagram (Fig. 4A) and the scatter plots examined through Wilcoxon signed-rank tests indicated that $\mathrm{T}$ classification, tumor stage, tumor grade, Child-Pugh grade, eastern cancer oncology group (ECOG) score, vascular invasion, and survival status (Fig. 4B-H) were significantly associated with risk. Subsequently, univariate Cox regression analysis indicated that there were significant differences in tumor stage $(p<0.001, \mathrm{HR}=1.627,95 \% \mathrm{CI}[1.218-2.173])$, vascular invasion $(p=0.007, \mathrm{HR}=1.737,95 \% \mathrm{CI}[1.161-2.599])$, ECOG $(p<0.001, \mathrm{HR}=1.945,95 \%$ CI $[1.346-2.811])$, and risk score $(p<0.001, \mathrm{HR}=1.028,95 \% \mathrm{CI}[1.017$
- 1.039]), while tumor stage $(p=0.018, \mathrm{HR}=1.488,95 \%$ CI $[1.072-2.067])$, ECOG $(p<0.001, \mathrm{HR}=1.905,95 \%$ CI $[1.299-2.792])$ and risk score $(p<0.001, \mathrm{HR}=1.024$, 95\% CI [1.015-1.034]) were determined to be independent prognostic predictors by multivariate Cox regression (Fig. 4I).

\section{Relationship between tumor-infiltrating immune cells, immune molecules and the risk model}

Since lncRNAs were initially associated with the irgene, we explored whether this model correlates with the tumor immune microenvironment. Our study found that there was a positive correlation between the highrisk group and tumor-infiltrating immune cells, such as $\mathrm{B}$ cells, neutrophils, and macrophages, but a negative correlation with $\mathrm{CD} 8+\mathrm{T}$ cells, CD4 $+\mathrm{T}$ cells, and monocytes. Spearman correlation analysis was carried out in detail, and the results are shown in a lollipop diagram (Fig. 5A). Since ICIs currently play significant roles in the treatment of $\mathrm{HCC}$, we explored the correlation between the risk model and ICI-related biomarkers. The results showed that high risk scores were associated with the expression of CD276 $(p<0.001)$, GSDME $(p<0.001)$, HAVCR2 $(p<0.01)$, and TNFRSF18 $(p<0.05)$ (Fig. 5B-E).

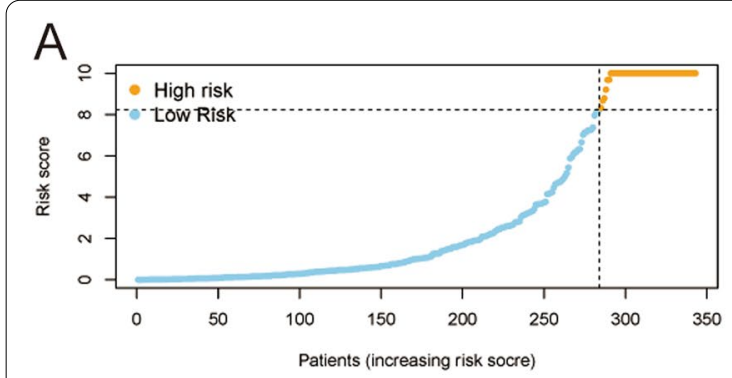

B
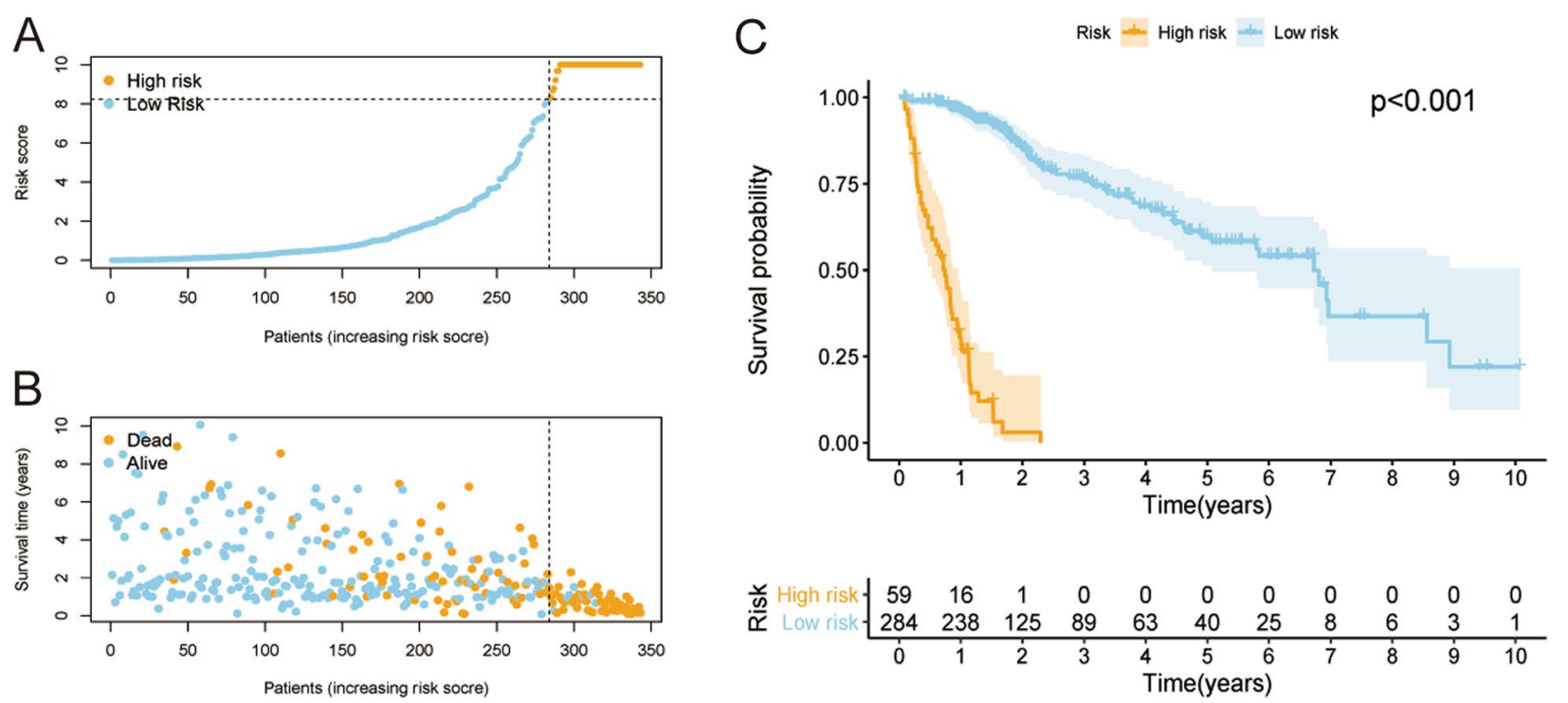

Fig. 3 Prognostic power of the risk assessment model. Risk score (A) and survival outcome (B) of each case. Kaplan-Meier survival curve of the high-risk group and low-risk group $(\mathbf{C})$

(See figure on next page.)

Fig. 4 Application of the risk assessment model to a clinical evaluation. A strip diagram (A) and scatter plot show that $T$ classification (B), tumor stage $(\mathbf{C})$, tumor grade $(\mathbf{D})$, Child-Pugh grade $(\mathbf{E})$, ECOG $(\mathbf{F})$, vascular invasion $(\mathbf{G})$, and survival status $(\mathbf{H})$ were significantly correlated with risk score. Univariate and multivariate Cox regression analyses were performed to analyze the clinicopathological features, and the results are shown in a forest $\operatorname{map}(\mathbf{I})$ 

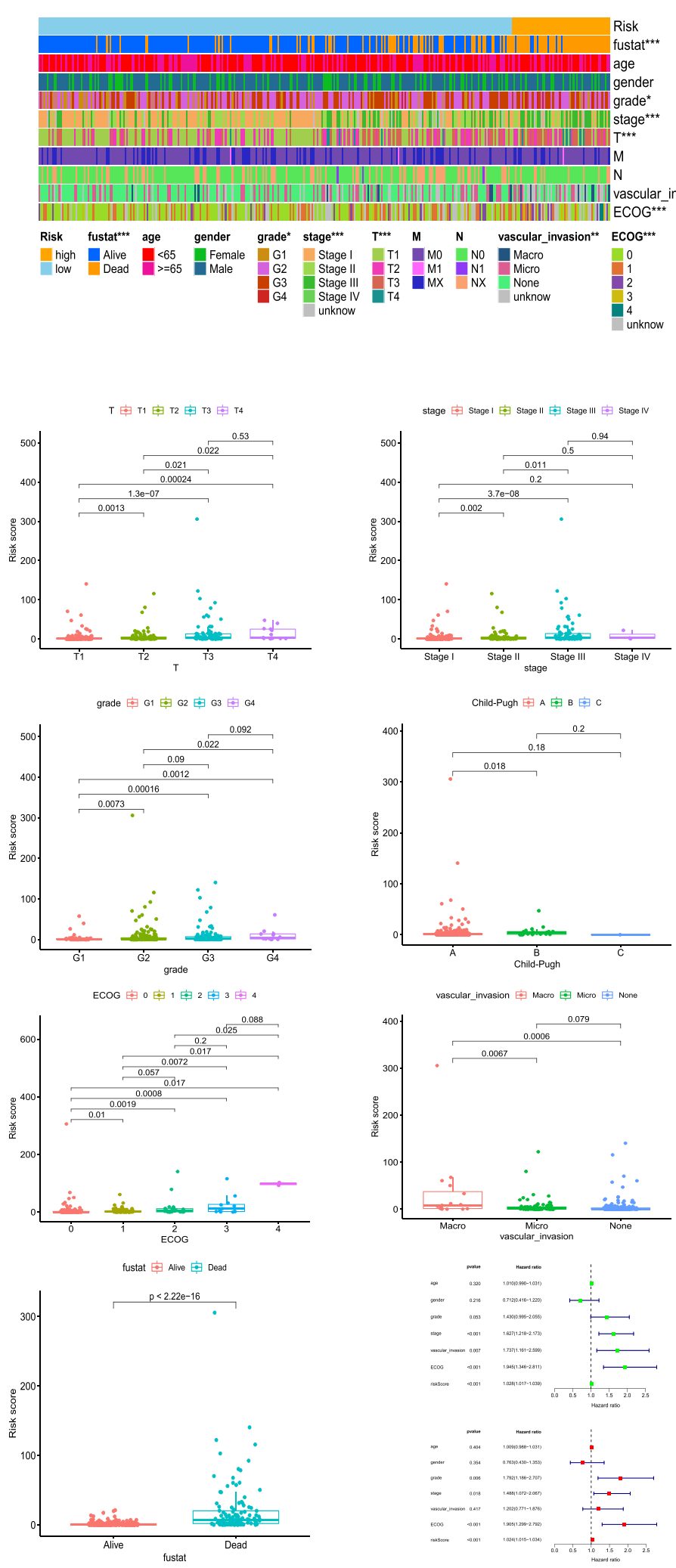

Fig. 4 (See legend on previous page.) 
There was no significant difference between the high-risk scores and ir-genes, such as CTLA4, PDCD1, and LAG3 (all $p>0.05$, Fig. 5F-H).

\section{Correlation analysis of the risk model and chemotherapy drugs}

In addition to checkpoint blocking therapy, we explored the association between the risk and efficacy of chemotherapy drugs commonly used for HCC. We found that the high-risk score was accompanied by a higher half inhibitory concentration (IC50) of chemotherapy drugs for erlotinib $(p<0.001)$, methotrexate $(p=0.0024)$, and rapamycin $(p=0.0043)$ and a lower IC50 for bleomycin $(p<0.001)$, doxorubicin $(p=0.0023)$, gemcitabine $(p<0.001)$, mitomycin $(p<0.001)$, and paclitaxel $(p=0.011)$, suggesting that this model can serve as a potential predictor for the sensitivity of chemotherapies (Fig. 6A-J).

\section{Discussion}

It is necessary to improve the accuracy of prognostic markers for HCC patients. LncRNAs are closely related to normal physiological activities and the development of diseases [10, 30]. Furthermore, studies have demonstrated that lncRNAs play vital roles in tumor development and antitumor processes [31-33]. Recent studies have focused on investigating the potential relationship between coding genes and noncoding RNAs to predict patient prognosis with cancers [20,34]. Unfortunately, the majority of these signatures were generated with the specific expression levels of transcripts. In our research, we ignored the specific expression levels of lncRNAs and utilized ir-gene pairing to generate a practical model with a combination of lncRNAs.

First, we downloaded the original information of lncRNAs from the TCGA database, and then a differential coexpression analysis was performed to catalog the DEirlncRNAs. The lncRNA pairs were verified by an improved cyclic single pair method along with 0 or 1 matrix. Second, univariate analysis and modified LASSO penalty regression were performed to determine DEirlncRNA pairs, procedures including cross-validation, multiple repetitions, and random stimulation. Then, we gained the optimum model by examining each AUC value of the ROC curve, and the optimum cutoff point was determined according to the AIC value of each point on the AUC to distinguish the high-risk and low-risk groups in the HCC dataset. Finally, the model was evaluated according to various parameters, such as survival rate, clinicopathological features, tumor-infiltrating immune cells, checkpoint-associated molecules, and chemotherapeutics.

The origin of lncRNAs may have the following four sources, mutation of a protein-coding gene, chromosomal rearrangement, duplications, and transposon insertion [35, 36]. Current research reveals that the phenotypic characteristics of lncRNAs regulation of cancer mainly include: cell proliferation, growth inhibition, cell migration, cell immortalization, angiogenesis, and cell viability [37]. The relationship between lncRNAs and tumors has received increasing attention [37-39]. Deng et al. established a model to predict HCC patient survival [40]. The method utilized in this study does require data on the specific expression level of each lncRNA; only pairs with high or low expression levels need to be detected. Therefore, the model is practical and straightforward in distinguishing high-risk or low-risk clinical cases. The lncRNAs included in this model are related to ir-genes; Therefore, these lncRNAs may regulate the immune microenvironment and the activation of immune cells.

Our research reveals that some of the DEirlncRNAs included in the modeling play vital roles in the malignant phenotype of many cancers, such as MYLK-AS1 [41, 42], THUMPD3 - AS1 [22], and DSCR8 [43], especially in the development of HCC. MYLK-AS1 promotes angiogenesis and HCC progression by targeting the miR424-5p/E2F7 axis and activating the VEGFR-2 signaling pathway [42]. THUMPD3-AS1 was associated with the cell cycle and can be used as a prognostic marker in hepatitis B virus-related HCC patients [22]. Wang et al. revealed that DSCR8 promotes the progression of HCC by activating the Wnt/b-catenin signaling pathway [44]. The established model can identify new biomarkers for further tumor-related studies.

To achieve better accuracy and effectiveness of risk prediction, this study used the improved method of the LASSO penalty model [45]. In addition, we determined the maximum value for an optimal model by calculating each AUC value and then compared it with other clinicopathological characteristics, further improving the modeling process. The AIC value was used to obtain the ideal cutoff point for model fitting; the median value was not used to discriminate risk. After using this new method

(See figure on next page.)

Fig. 5 Estimation of tumor-infiltrating cells and immunosuppressive molecules with the risk assessment model. A Correlation between the high-risk group and tumor-infiltrating immune cells. High-risk scores were associated with the expression of CD276 (B), GSDME (C), HAVCR2 (D), and TNFRSF18 (E). There was no significant difference between the high-risk scores and the expression of immune-related genes, such as CTLA4 (F), $\operatorname{PDCD1}(\mathbf{G})$, and LAG3 (H) 

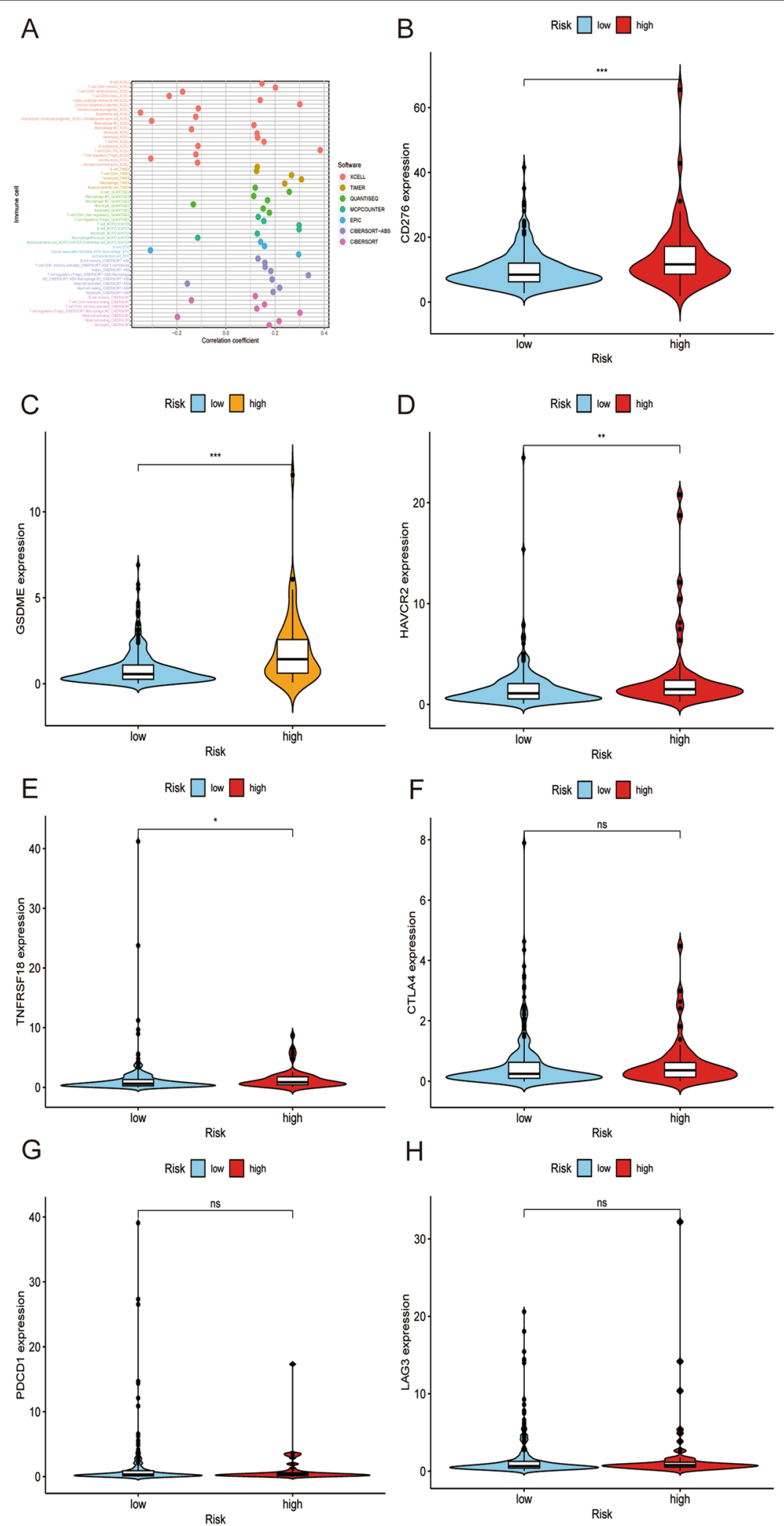

Fig. 5 (See legend on previous page.) 

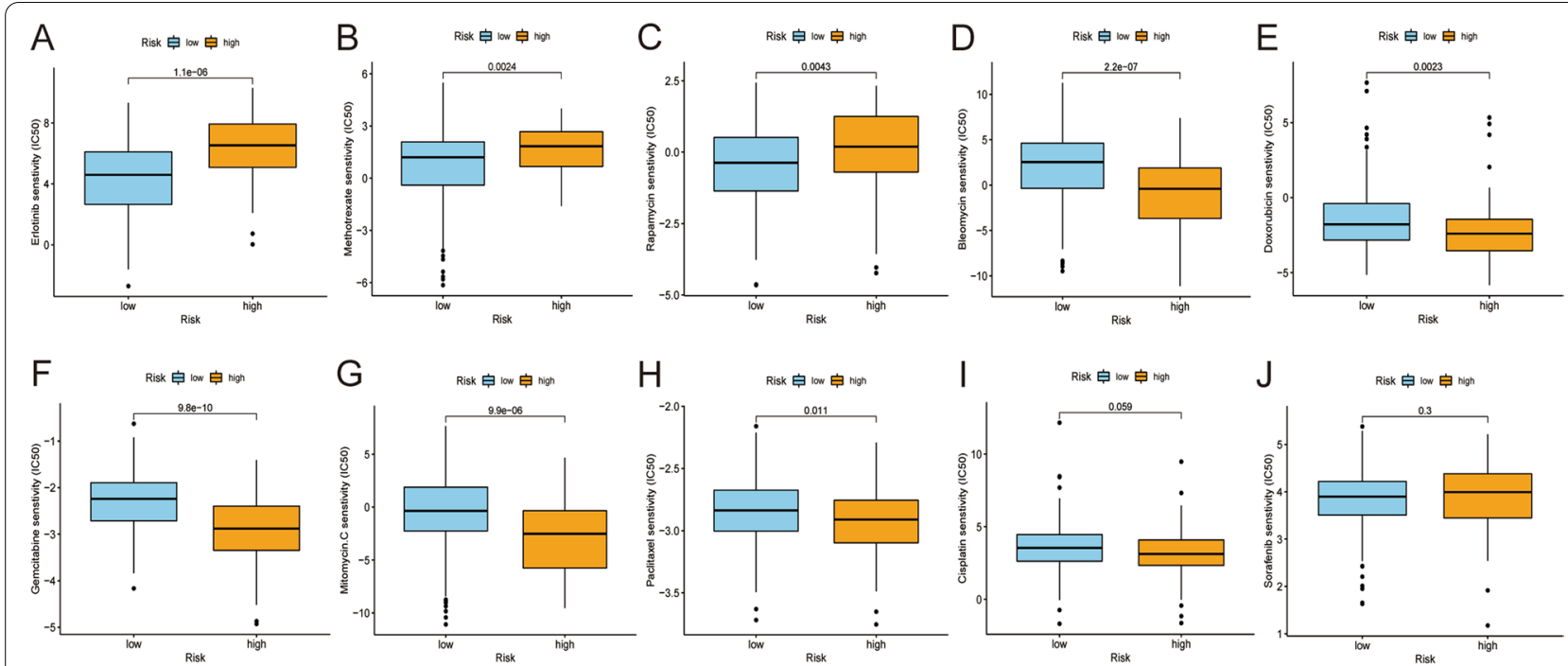

Fig. 6 Relationship between risk scores and the IC50 of chemotherapeutics

to differentiate high-risk and low-risk groups, survival outcomes and univariate and multivariate analyses of clinicopathological features were reevaluated. Moreover, the sensitivity of chemotherapy drugs commonly used to treat HCC treatment was analyzed. The relationship between high-risk and low-risk groups and immune cell infiltration into tumors and the relationship between high-risk and low-risk groups and immune checkpointrelated genes were also studied, and the results indicated that this modeling algorithm has a good clinical application prospects.

The immune checkpoint blockade reaction is closely related to tumor-infiltrating immune cells [46]. Our research used seven commonly recognized methods to identify infiltrating immune cell to investigate the relationship between risk scores and tumor-infiltrating immune cells, including XCELL [47, 48], TIMER [49, 50], QUANTISEQ [51, 52], MCPCOUNTER [53], EPIC [54], CIBERSORT-ABS, and CIBERSORT $[55,56]$. Due to the defects and complexity of these algorithms, they are rarely compared with each other. Through integration analysis, our findings show that DEirlncRNA pairs have a positive correlation with tumor-infiltrating immune cells such as B cells, neutrophils, and macrophages but are negatively correlated with $\mathrm{CD} 8+\mathrm{T}$ cells, CD4+ T cells, and monocytes. Wang et al. demonstrated that the immune score can predict the efficacy of immunotherapy and chemotherapy [57]. IrlncRNA SATB2-AS1 can affect the tumor immune cell microenvironment and inhibit colorectal cancer metastasis [41]. LncRNA-EGFR can stimulate $\mathrm{T}$ regulatory cell differentiation and promote immune evasion in HCC [42]. Our model suggests that high risk is related to sensitivity to chemotherapy drugs such as methotrexate, rapamycin, bleomycin, doxorubicin, gemcitabine, mitomycin, and paclitaxel but not sensitivity to sorafenib. We believe that immunotherapy is more effective than traditional chemotherapy, mainly because immunotherapy can activate immune cell functions and promote tumor resistance by triggering active immunity. Tumor mutations can cause a large number of neoantigens to be released, which can be recognized by $T$ cells and cause many immune cells to infiltrate into tumors [58-60].

We acknowledge that our study has some limitations. First, the research data were based on public databases. Some data were incomplete, such as some clinicopathological features and the sensitivity of drugs commonly used in the treatment of $\mathrm{HCC}$; for instance, lenvatinib and oxaliplatin have not been analyzed. Second, the constructed model needs external verification because the expression level of each sample differs, which may lead to an unreliable final model. Third, this study did not analyze the expression level of these lncRNAs in immune cells. However, this research uses various methods to verify the new modeling algorithm and optimizes and analyzes it. Despite the lack of external data validation, from the analysis results, our model was acceptable. However, this study will be more convincing when external validation is performed. Therefore, our team will recollect clinicopathological data for subsequent studies and enlarge the sample size for further verification. 


\section{Conclusions}

Our research shows that an innovative signature established by irlncRNAs that does not require data on the expression levels of lncRNAs to predict HCC patient prognosis and may contribute to identification of patients who can benefit from antitumor immunotherapy.

\begin{abstract}
Abbreviations
HCC: Hepatocellular carcinoma; TCGA: The Cancer Genome Atlas; IncRNAs: Long non-coding RNAs; irIncRNA: Immune-related long non-coding ribonucleic acids; DEirlncRNA: Different expression irlncRNA; miRNA: microRNA; LASSO: Least absolute shrinkage and selection operator; AUC: Area under the ROC curve; AIC: Akaike information criterion; ROC: Receiver operating characteristic; ICls: Immune checkpoint inhibitors; FPKM: Fragments perkilobase million; ir-genes: Immune-related genes; FC: Fold change; FDR: False discovery rate; GTF: Gene transfer format; ECOG: Eastern cancer oncology group; IC50: Half inhibitory concentration.
\end{abstract}

\section{Supplementary Information}

The online version contains supplementary material available at https://doi. org/10.1186/s12885-021-09059-x.

\section{Additional file 1.}

Additional file 2.

Additional file 3.

Additional file 4.

\section{Acknowledgements}

We acknowledge TCGA database for providing its platform and contributors for uploading their meaningful datasets.

\section{Statement}

The pre-print version of this article is present on https://www.researchsquare. com/article/rs-412155/v1. This article is not published nor is under publication elsewhere.

\section{Authors' contributions}

Conceptualization, project administration, formal analysis, software, and writing - review and editing, M.D.; Data analysis, software, figures preparation, J.-B.L.; Data curation and analysis, figures, and tables preparation, R.-C.Z., W.-P.L.; Data collection and analysis, S.-H.L. J.-W.Z.; Conceived and designed this study, W.W.; Conceptualization, supervision, writing-review, and editing, R.-P.G. All authors have read and approved the final version of the manuscript.

\section{Funding}

This study was supported by the National Natural Science Foundation of China (No. 81871985); Natural Science Foundation of Guangdong Province (No. 2018A0303130098); Science and Technology Planning Project of Guangdong Province (No. 2017A020215112); Science and Technology Planning Project of Guangzhou (No. 201903010017 and No. 201904010479); and Medical Innovation Project of the First Affiliated Hospital of Guangzhou University of Chinese Medicine (2019IIT18). The funding bodies played no role in the design of the study and collection, analysis, and interpretation of data and in writing the manuscript.

\section{Availability of data and materials}

Publicly available datasets were analyzed in this study, these can be found in The Cancer Genome Atlas (https://portal.gdc.cancer.gov/). Supplementary data associated with this article, named "Table S1", "Table S2", "Table S3", and "Fig. S1A" have been uploaded.

\section{Declarations}

Ethics approval and consent to participate

This article does not contain any studies with human participants or animals performed by any of the authors.

\section{Consent for publication}

Not applicable.

\section{Competing interests}

We declare that all authors do not have competing interests related to this research project.

\section{Author details}

${ }^{1}$ Department of Liver Surgery, Sun Yat-sen University Cancer Center, Guangzhou, China. ${ }^{2}$ State Key Laboratory of Oncology in South China, Guangzhou, China. ${ }^{3}$ Collaborative Innovation Center for Cancer Medicine, 651 Dongfeng East Road, Guangzhou, China. ${ }^{4}$ Department of Health Management Center, Nanfang Hospital, Southern Medical University, Guangzhou, China.

Received: 30 May 2021 Accepted: 28 November 2021

Published online: 19 December 2021

\section{References}

1. Villanueva A. Hepatocellular Carcinoma. N Engl J Med. 2019:380(15):1450-62.

2. Bray F, Ferlay J, Soerjomataram I, Siegel RL, Torre LA, Jemal A. Global cancer statistics 2018: GLOBOCAN estimates of incidence and mortality worldwide for 36 cancers in 185 countries. CA Cancer J Clin. 2018;68:394-424.

3. Forner A, Reig M, Bruix J. Hepatocellular carcinoma. Lancet. 2018;391:1301-14.

4. Havel JJ, Chowell D, Chan TA. The evolving landscape of biomarkers for checkpoint inhibitor immunotherapy. Nat Rev Cancer. 2019;19:133-50.

5. El-Khoueiry AB, Sangro B, Yau T, Crocenzi TS, Kudo M, Hsu C, et al. Nivolumab in patients with advanced hepatocellular carcinoma (CheckMate 040): an open-label, non-comparative, phase 1/2 dose escalation and expansion trial. Lancet. 2017;389:2492-502.

6. Finn RS, Qin S, Ikeda M, Galle PR, Ducreux M, Kim TY, et al. Atezolizumab plus Bevacizumab in Unresectable Hepatocellular Carcinoma. N Engl J Med. 2020;382:1894-905

7. Zhu AX, Finn RS, Edeline J, Cattan S, Ogasawara S, Palmer D, et al. Pembrolizumab in patients with advanced hepatocellular carcinoma previously treated with sorafenib (KEYNOTE-224): a non-randomised, open-label phase 2 trial. Lancet Oncol. 2018;19:940-52.

8. Qian X, Zhao J, Yeung PY, Zhang QC, Kwok CK. Revealing IncRNA structures and interactions by sequencing-based approaches. Trends Biochem Sci. 2019;44:33-52.

9. Yang G, Lu X, Yuan L. LncRNA: a link between RNA and cancer. Biochim Biophys Acta. 2014;1839:1097-109.

10. Sun X, Zheng H, Sui N. Regulation mechanism of long non-coding RNA in plant response to stress. Biochem Biophys Res Commun. 2018:503:402-7.

11. Quinn JJ, Chang HY. Unique features of long non-coding RNA biogenesis and function. Nat Rev Genet. 2016;17:47-62.

12. Robinson EK, Covarrubias S, Carpenter S. The how and why of IncRNA function: an innate immune perspective. Biochim Biophys Acta Gene Regul Mech. 2020;1863:194419.

13. Zhao J, Lawless MW. Long noncoding RNAs and their role in the liver cancer axis. Nat Rev Gastroenterol Hepatol. 2013;10:703.

14. Schmitt AM, Chang HY. Long noncoding RNAs in Cancer pathways. Cancer Cell. 2016;29:452-63.

15. Atianand MK, Caffrey DR, Fitzgerald KA. Immunobiology of long noncoding RNAs. Annu Rev Immunol. 2017;35:177-98.

16. Chen YG, Satpathy AT, Chang HY. Gene regulation in the immune system by long noncoding RNAs. Nat Immunol. 2017;18:962-72. 
17. Peng D, Wang L, Li H, Cai C, Tan Y, Xu B, et al. An immune infiltration signature to predict the overall survival of patients with colon cancer. IUBMB Life. 2019;71:1760-70.

18. Wei ZW, Wu J, Huang WB, Li J, Lu XF, Yuan YJ, et al. Immune-infiltration based signature as a novel prognostic biomarker in gastrointestinal stromal tumour. EBioMedicine. 2020;57:102850.

19. Zhang B, Wang Q, Fu C, Jiang C, Ma S. Exploration of the immune-related signature and immune infiltration analysis for breast ductal and lobular carcinoma. Ann Transl Med. 2019;7:730.

20. Qu L, Wang ZL, Chen Q, Li YM, He HW, Hsieh JJ, et al. Prognostic value of a long non-coding RNA signature in localized clear cell renal cell carcinoma. Eur Urol. 2018;74:756-63.

21. Li Z, Zhang J, Liu X, Li S, Wang Q, Chen D, et al. The LINC01138 drives malignancies via activating arginine methyltransferase 5 in hepatocellular carcinoma. Nat Commun. 2018;9:1572.

22. Zhao X, Bai Z, Li C, Sheng C, Li H. Identification of a novel eight-IncRNA prognostic signature for HBV-HCC and analysis of their functions based on Coexpression and ceRNA networks. Biomed Res Int. 2020;2020:8765461.

23. Zhou P, Lu Y, Zhang Y, Wang L. Construction of an immune-related sixIncRNA signature to predict the outcomes, immune cell infiltration, and immunotherapy response in patients with hepatocellular carcinoma. Front Oncol. 2021;11:661758.

24. Wu HC, Yang HI, Wang Q, Chen CJ, Santella RM. Plasma DNA methylation marker and hepatocellular carcinoma risk prediction model for the general population. Carcinogenesis. 2017;38:1021-8.

25. Lv Y, Lin SY, Hu FF, Ye Z, Zhang Q, Wang Y, et al. Landscape of cancer diagnostic biomarkers from specifically expressed genes. Brief Bioinformatics. 2020:21:2175-84

26. Zhang $Y$, Zhang L, Xu Y, Wu X, Zhou Y, Mo J. Immune-related long noncoding RNA signature for predicting survival and immune checkpoint blockade in hepatocellular carcinoma. J Cell Physiol. 2020;235:9304-16.

27. Wu Y, Wang L, Wang X, Zhao Y, Mao A, Zhang N, et al. RNA sequencing analysis reveals the competing endogenous RNAs interplay in resected hepatocellular carcinoma patients who received interferon-alpha therapy. Cancer Cell Int. 2021;21:464.

28. Hong W, Liang L, Gu Y, Qi Z, Qiu H, Yang X, et al. Immune-related IncRNA to construct novel signature and predict the immune landscape of human hepatocellular carcinoma. Mol Ther Nucleic Acids. 2020;22:937-47

29. Yin J, Li X, LV C, He X, Luo X, Li S, et al. Immune-related IncRNA signature for predicting the immune landscape of head and neck squamous cell carcinoma. Front Mol Biosci. 2021;8:689224.

30. Zhu J, Fu H, Wu Y, Zheng X. Function of IncRNAs and approaches to IncRNA-protein interactions. Sci China Life Sci. 2013;56:876-85.

31. Li J, Meng H, Bai Y, Wang K. Regulation of IncRNA and its role in Cancer metastasis. Oncol Res. 2016;23:205-17.

32. Huang Z, Zhou JK, Peng Y, He W, Huang C. The role of long noncoding RNAs in hepatocellular carcinoma. Mol Cancer. 2020;19:77.

33. Sanchez Calle A, Kawamura Y, Yamamoto Y, Takeshita F, Ochiya T. Emerging roles of long non-coding RNA in cancer. Cancer Sci. 2018;109:2093-100.

34. Zhu X, Tian X, Yu C, Shen C, Yan T, Hong J, et al. A long non-coding RNA signature to improve prognosis prediction of gastric cancer. Mol Cancer. 2016;15:60.

35. Alessio E, Bonadio RS, Buson L, Chemello F, Cagnin S. A single cell but many different transcripts: a journey into the world of long non-coding RNAs. Int J Mol Sci. 2020;21(1):302

36. Wang KC, Chang HY. Molecular mechanisms of long noncoding RNAs. Mol Cell. 2011:43:904-14.

37. Bhan A, Soleimani M, Mandal SS. Long noncoding RNA and Cancer: a new paradigm. Cancer Res. 2017;77:3965-81.

38. Chan JJ, Tay Y. Noncoding RNA:RNA regulatory networks in Cancer. Int J Mol Sci. 2018:19(5):1310.

39. Renganathan A, Felley-Bosco E. Long noncoding RNAs in Cancer and therapeutic potential. Adv Exp Med Biol. 2017:1008:199-222.

40. Deng B, Yang M, Wang M, Liu Z. Development and validation of 9-long non-coding RNA signature to predicting survival in hepatocellular carcinoma. Medicine (Baltimore). 2020;99:e20422
41. Xu M, Xu X, Pan B, Chen X, Lin K, Zeng K, et al. LncRNA SATB2-AS1 inhibits tumor metastasis and affects the tumor immune cell microenvironment in colorectal cancer by regulating SATB2. Mol Cancer. 2019;18:135.

42. Jiang R, Tang J, Chen Y, Deng L, Ji J, Xie Y, et al. The long noncoding RNA Inc-EGFR stimulates T-regulatory cells differentiation thus promoting hepatocellular carcinoma immune evasion. Nat Commun. 2017;8:15129.

43. You Q, Yao Y, Wu J, Cheng C, Li Y, Yuan H. YY1-induced IncRNA DSCR8 promotes the progression of ovarian cancer via miR-3192-5p/YY1 axis. Biomed Pharmacother. 2020;129:110339.

44. Wang Y, Sun L, Wang L, Liu Z, Li Q, Yao B, et al. Long non-coding RNA DSCR8 acts as a molecular sponge for miR-485-5p to activate Wnt/ $\beta$ catenin signal pathway in hepatocellular carcinoma. Cell Death Dis. 2018;9:851.

45. Sveen A, Ågesen TH, Nesbakken A, Meling Gl, Rognum TO, Liestøl K, et al. ColoGuidePro: a prognostic 7-gene expression signature for stage III colorectal cancer patients. Clin Cancer Res. 2012;18:6001-10.

46. Zhou G, Sprengers D, Boor P, Doukas M, Schutz H, Mancham S, et al. Antibodies against immune checkpoint molecules restore functions of tumor-infiltrating T cells in hepatocellular carcinomas. Gastroenterology. 2017:153:1107-1119.e10.

47. Aran D. Cell-type enrichment analysis of bulk Transcriptomes using xCell. Methods Mol Biol. 2020;2120:263-76.

48. Aran D, Hu Z, Butte AJ. xCell: digitally portraying the tissue cellular heterogeneity landscape. Genome Biol. 2017;18:220.

49. Li T, Fan J, Wang B, Traugh N, Chen Q, Liu JS, et al. A web server for comprehensive analysis of tumor-infiltrating immune cells. Cancer Res. 2017:77:e108-108e110.

50. Li T, Fu J, Zeng Z, Cohen D, Li J, Chen Q, et al. TIMER2.0 for analysis of tumor-infiltrating immune cells. Nucleic Acids Res. 2020;48:W509-509W514.

51. Finotello F, Mayer C, Plattner C, Laschober G, Rieder D, Hackl H, et al. Molecular and pharmacological modulators of the tumor immune contexture revealed by deconvolution of RNA-seq data. Genome Med. 2019;11:34.

52. Plattner C, Finotello F, Rieder D. Deconvoluting tumor-infiltrating immune cells from RNA-seq data using quanTIseq. Meth Enzymol. 2020;636:261-85

53. Dienstmann R, Villacampa G, Sveen A, Mason MJ, Niedzwiecki D, Nesbakken A, et al. Relative contribution of clinicopathological variables, genomic markers, transcriptomic subtyping and microenvironment features for outcome prediction in stage II/III colorectal cancer. Ann Oncol. 2019:30:1622-9.

54. Racle J, de Jonge K, Baumgaertner P, Speiser DE, Gfeller D. Simultaneous enumeration of cancer and immune cell types from bulk tumor gene expression data. Elife. 2017;6:e26476

55. Zhang H, Li R, Cao Y, Gu Y, Lin C, Liu X, Lv K, He X, Fang H, Jin K, et al. Poor clinical outcomes and Immunoevasive contexture in Intratumoral IL10-producing macrophages enriched gastric Cancer patients. Ann Surg. 2020. https://doi.org/10.1097/SLA.0000000000004037.

56. Chen B, Khodadoust MS, Liu CL, Newman AM, Alizadeh AA. Profiling tumor infiltrating immune cells with CIBERSORT. Methods Mol Biol. 2018;1711:243-59.

57. Dai GP, Wang LP, Wen YQ, Ren XQ, Zuo SG. Identification of key genes for predicting colorectal cancer prognosis by integrated bioinformatics analysis. Oncol Lett. 2020;19:388-98.

58. Schumacher TN, Schreiber RD. Neoantigens in cancer immunotherapy. Science. 2015;348:69-74.

59. Chandran SS, Klebanoff CA. T cell receptor-based cancer immunotherapy: emerging efficacy and pathways of resistance. Immunol Rev. 2019:290:127-47.

60. Yamamoto TN, Kishton RJ, Restifo NP. Developing neoantigen-targeted T cell-based treatments for solid tumors. Nat Med. 2019:25:1488-99.

\section{Publisher's Note}

Springer Nature remains neutral with regard to jurisdictional claims in published maps and institutional affiliations. 